\title{
Relative distance cues contribute to scaling depth from motion parallax
}

\author{
SATOKO OHTSUKA \\ University of Tokyo, Tokyo, Japan \\ and \\ HIROYASU UJIKE and SHINYA SAIDA \\ National Institute of Advanced Industrial Science and Technology, Tsukuba, Japan
}

\begin{abstract}
The visual system scales motion parallax signals with information about absolute distance (M. E. Ono, Rivest, \& H. Ono, 1986). The present study was designed to determine whether relative distance cues, which intrinsically provide information about relative distance, contribute to this scaling. In two experiments, two test stimuli, containing an equal extent of motion parallax, were presented simultaneously at a fixed viewing distance. The relative distance cues of dynamic occlusion and motion parallax in the areas surrounding the test stimuli (background motion parallax) and/or relative size were manipulated. The observers reported which of the two parallactic test stimuli appeared to have greater depth, and which appeared to be more distant. The results showed that the test stimulus specified, by the relative distance cues, as being more distant was perceived as having more depth and as being more distant. This indicates that relative distance cues contribute to scaling depth from motion parallax by modifying the information about the absolute distance of objects.
\end{abstract}

Although it is widely accepted that information about the absolute distance ${ }^{1}$ of a visual object is obtained from absolute distance cues (Gillam, 1995), it is possible that this information is modified by relative distance cues. For example, it has been suggested that relative distance cues, which primarily provide information about the relative orders and/or ratios among object distances, can influence observers' estimates of absolute distance (Foley \& Held, 1972; Gogel, 1972). Specifically, Foley and Held had observers point to a visual target with their unseen hand in two conditions: A convergence condition in which only convergence information was available, and a multicue condition in which the cues of relative size, binocular disparity, perspective, accommodation, and convergence were available. They found that the observers' estimates of absolute distance were more accurate and precise in the multicue condition than in the convergence alone condition.

If, as suggested above, relative distance cues do indeed modify absolute distance information, it follows that they should also affect scaling depths perceived from motion parallax - that is, relative motion disparities yoked to the observer's head movement (Rogers \& Graham, 1979; van

This research was supported by a JSPS Research Fellowship for Young Scientists awarded to the first author. The authors thank G. J. Andersen, H. A. Sedgwick, and the anonymous reviewers for their valuable comments and suggestions on the original manuscript. We also thank Alistair P. Mapp for helpful comments on our logic and English. Correspondence concerning this article should be addressed to S. Ohtsuka, Intelligent Modeling Laboratory, University of Tokyo, 2-11-16 Yayoi, Bunkyo-ku,Tokyo 113-8656,Japan (e-mail: sohtsuka@iml.u-tokyo.ac.jp).
Damme, Oosterhoff, \& van de Grind, 1994). This is so, because, as is shown in Figure 1, the magnitude of depth specified by a given extent of motion parallax increases with the absolute distance (M. E. Ono, Rivest, \& H. Ono, 1986). Accordingly, it is geometrically expected that the visual system scales the depth information from motion parallax with the absolute distance. Psychophysical data have confirmed this (Ohtsuka, Ujike, \& Saida, 1999; M. E. Ono et al., 1986). M. E. Ono et al., for example, reported that surfaces defined by equal extents of motion parallax were perceived to have greater depth when viewed from a distance of $80 \mathrm{~cm}$ than when viewed from $40 \mathrm{~cm}$.

Thus, the primary purpose of the present study was to determine whether relative distance cues contribute to scaling depth perceived from motion parallax. We conducted two experiments in which we manipulated the relative distance cues associated with two identical and simultaneously presented parallactic test stimuli. If relative distance cues contribute to the scaling of parallactic depth, the test stimulus specified as being at a relatively farther distance would be perceived as having greater depth and as being at a farther distance than the other test stimulus.

The secondary purpose of the present study was to determine whether combined relative distance cues have a greater effect on the scaling of parallactic depth than do the individual cues alone. It is well known that the perception of depth is more accurate when multiple depth cues are combined than when only one cue is available (Bülthoff \& Mallot, 1988; Curran \& Johnston, 1994; Johnston, Cumming, \& Landy, 1994; Rogers \& Collett, 1989). Similarly, the perception of absolute distance is more accurate when 


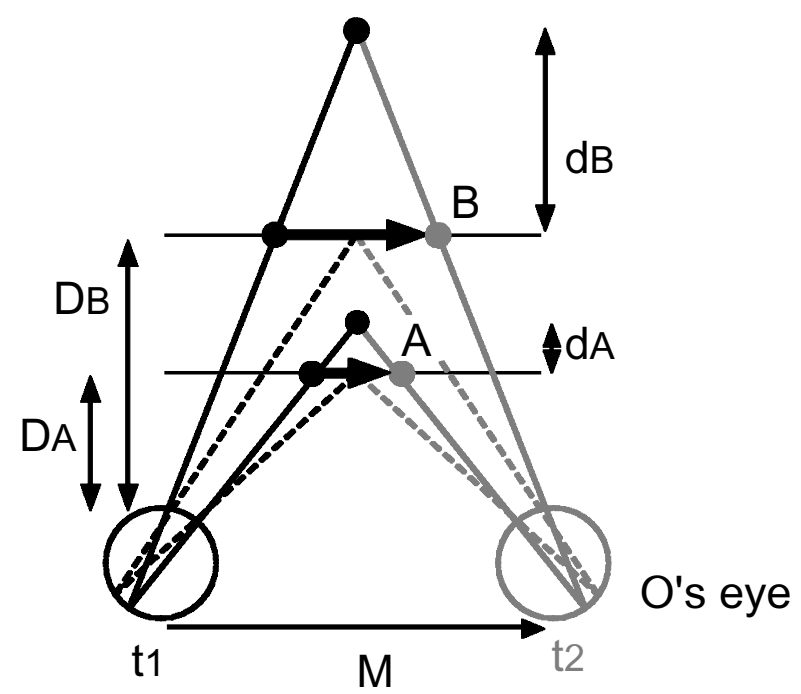

Figure 1. An illustration showing that, geometrically, the magnitude of depth (dA and $\mathrm{dB}$ ) specified by an equal extent of motion parallax increases with absolute distance from the observer to the object (DA and DB). In this figure, the observer's head (indicated by an eye) moves through the extent of $M$ between $t 1$ and $t 2$, and the motion disparity of $A$ and $B$ is the same.

multiple absolute distance cues are combined (Künnapas, 1968; Swenson, 1932). Thus, it is likely that the combined effect of multiple relative distance cues on the scaling of parallactic depth, as well as on the information about absolute distance, will exceed the effect of individual cues. To examine this issue, we manipulated multiple relative distance cues associated with two identical and simultaneously presented parallactic test stimuli and compared the perceived depths and distances of the test stimuli with those obtained when the individual relative distance cues were manipulated.

\section{EXPERIMENT 1}

Experiment 1 was designed to determine whether relative distance cues contribute to scaling depth from motion parallax. The relative distance cues examined were dynamic occlusion and relative motion parallax. Dynamic occlusion is the appearance and disappearance of portions of distant surfaces resulting from the relative motion of less distant surfaces (Andersen \& Braunstein, 1983; Kaplan, 1969) and unambiguously specifies the relative distance order of overlapping surfaces. Given that the test stimuli were two identical motion parallax stimuli, our displays contained two types of motion parallax: one specifying the depth surface of the test stimuli (test motion parallax) and the other manipulated as a relative distance cue (background motion parallax).

\section{Method}

Stimuli. The stimulus display, which was $11.5^{\circ}$ (height) $\times 15.1^{\circ}$ (width) and which included a pair of vertically arranged test stimuli of $4.8^{\circ} \times 3.1^{\circ}$ each, was presented on a CRT monitor (Figure 2). The background area surrounding the test stimuli was vertically sepa- rated into two equal areas by a $0.05^{\circ}$ thick horizontal line. The individual test stimuli were located in the middle of each background area, and thus they were separated vertically by $1.0^{\circ}$. The entire display, including both the test stimuli and the background areas, were composed of random dots. Each dot was defined by one cycle of a horizontal sinusoidal luminance gradient with $14.9 \mathrm{~cd} / \mathrm{m}^{2}$ at its peak and $99.7 \%$ contrast. Dot size was $0.05^{\circ} \times 0.15^{\circ}$, and dot density was $50 \%$. The test stimuli were enclosed by a $0.05^{\circ}$ thick contour in order to separate them from their background areas.

Test stimuli. The two test stimuli simulated identical threedimensional surfaces through the use of motion parallax (test motion parallax). Each stimulus was composed of two cycles of a vertical sinusoidal corrugation in depth with the top, middle, and bottom sections protruding (see Figure 3 for a stereoscopic demonstration of the depth surface). This was achieved by yoking the horizontal motion of the dots in the test stimuli to the observers' lateral head movements of 10.0-cm extent, in such a way that the motion of the dots formed a velocity gradient of two cycles of a horizontal sinusoidal function. The extent of the test motion parallax was 4.0 arc min in equivalent disparity (Rogers \& Graham, 1982), corresponding to a peak-to-trough depth of $2.4 \mathrm{~cm}$. The spatial frequency of the depth corrugation was $0.42 \mathrm{cyc} / \mathrm{deg}$.

Background motion parallax. The relative distance cue of background motion parallax was produced by yoking the motion of the dots in the areas surrounding the test stimuli to the observers' lateral head movements (see Figure 2). Specifically, the dots in one of the background areas (e.g., the upper area) moved in the same direction as the observers' heads, whereas the dots in the other background area (e.g., the lower area) moved in the opposite direction. In both of the background areas, the dots moved 18.4 arc min while the observers' heads moved the extent of $10.0 \mathrm{~cm}$, which corresponded to 23.9 arc $\min$ in equivalent disparity and to $14.2 \mathrm{~cm}$ of depth.

Dynamic occlusion. The relative distance cue of dynamic occlusion was created by making use of the dot motions in the background areas described above. The test stimulus, experimentally defined as the front stimulus, dynamically occluded the background area and thus simulated a depth surface in front of the background (the upper
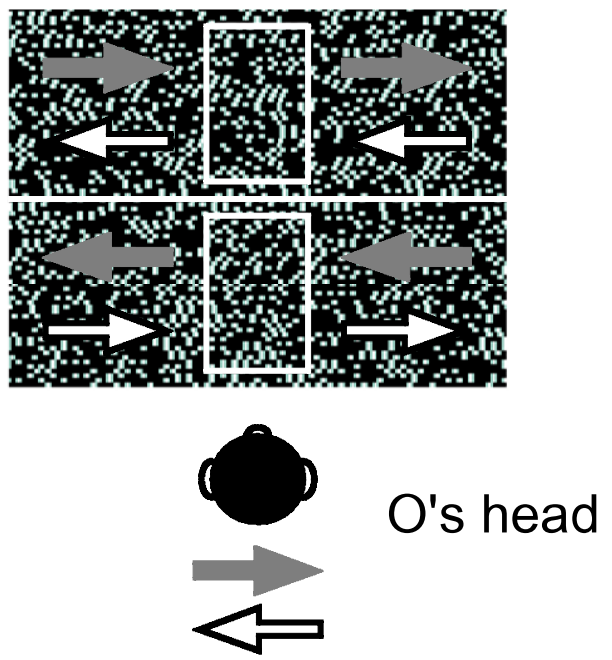

Figure 2. An illustration similar to the stimulus displays used in Experiment 1. The gray and white arrows schematically show an example of the background motions that generated the background motion parallax. These arrows show the direction of the background motion relative to the observer's head movement. The direction of the background motion relative to the observer's head movement was determined by the stimulus condition. 

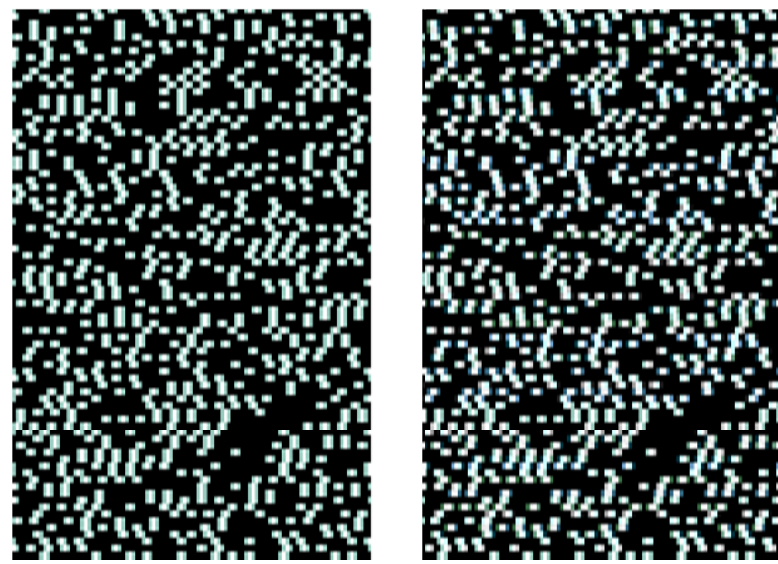

Figure 3. A stereoscopic demonstration of the depth surface simulated by each test stimulus with the use of test motion parallax. When viewed with uncrossed fusion, it appears to have two cycles of horizontal sinusoidal depth corrugations with the top, middle, and bottom sections protruding.

stimulus in Figure 4a). The other test stimulus, experimentally defined as the back stimulus, was dynamically occluded by the background area and thus simulated a depth surface behind the background (the lower stimulus in Figure 4a).

These dynamic occlusion conditions were achieved as follows. The front test stimulus was made to dynamically occlude the moving background area by making the background dots entering the contoured area of the front stimulus disappear and the background dots exiting the area reappear. During the background motion, the contour, as well as the average position of the random dots in the front stimulus, remained fixed on the CRT. When viewed with lateral head movement, the front stimulus appeared to be in front of the moving background. For the back test stimulus, the contour enclosing the back stimulus area moved concomitantly with the background motion without changing its rectangular shape. As with the front stimulus, the average position of the random dots in the back stimulus remained fixed on the CRT. The back stimulus was dynamically occluded by the moving background area by making the back stimulus dots exiting the contoured stimulus area disappear and the back stimulus dots entering the contoured stimulus area reappear. A visible portion of the back stimulus, therefore, shifted continuously yoked to the observers' head movements. When viewed with lateral head movement, the back stimulus appeared to be behind the moving background (i.e., it appeared to be lying behind a rectangular window that was moving with the background area). Figure $4 \mathrm{~b}$ demonstrates the manipulation of the dynamic occlusion stereoscopically.

We created the dynamic occlusion cue by fixing the test stimuli on the CRT and by making use of the motion of the background dots for the following reasons. First, fixing the test stimuli on the CRT ensured that the absolute distance cue of absolute motion parallax of the two test stimuli remained identical and constant during the observers' head movements. Second, by producing dynamic occlusion between the test stimuli and their background areas rather than between the two test stimuli, we ensured that the size and shape of the two test stimuli remained identical and constant.

Stimulus conditions. The two relative distance cues-background motion parallax and dynamic occlusion-were combined differentially to produce two stimulus conditions. In one condition, the background area surrounding the front stimulus moved in the opposite direction to the observers' heads, whereas the background area surrounding the back stimulus moved in the same direction (the upper panels in Figure 5). Thus, the background motion parallax in this condition specified that the background area surrounding the front stimulus was in front of the background area surrounding the back stimulus. When viewed with lateral head movement, the front stimulus often appeared in front of the back stimulus in this condition. Therefore, in this condition, which was named the enhancement condition, the background motion parallax emphasized or enhanced the difference in the relative distance between the front and back stimulus specified by the dynamic occlusion. If one assumes that these relative distance cues contribute to scaling parallactic depth by modifying information about absolute distance, it would be expected that both the perceived depth and the perceived distance of the back stimulus would exceed those of the front stimulus.

In the other condition, the background area surrounding the front stimulus moved in the same direction as the observers' heads, whereas the background area surrounding the back stimulus moved in the opposite direction (the lower panels in Figure 5). Thus, the background motion parallax in this condition specified that the background area surrounding the front stimulus was behind the background area sur-

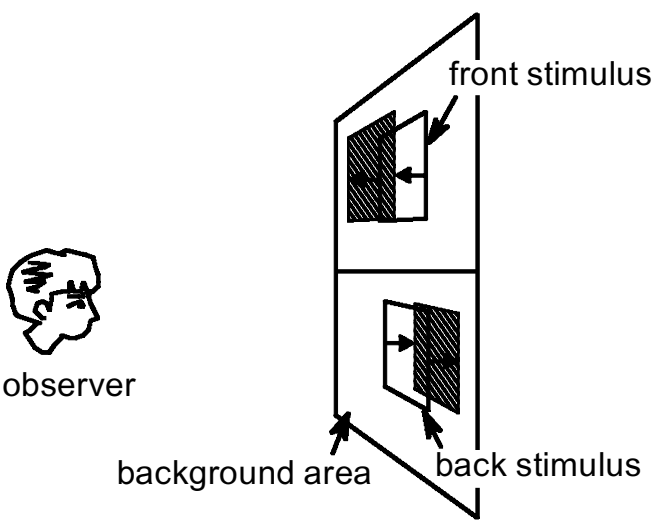

(a)

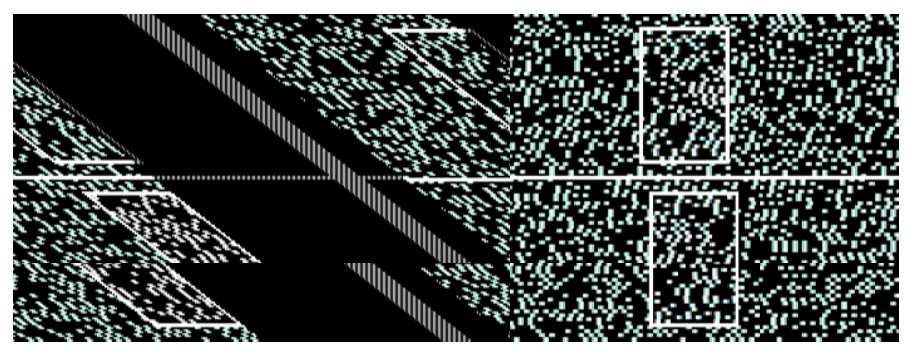

(b)

Figure 4. Illustrations demonstrating the manipulation of dynamic occlusion. In (a) and (b), the upper and lower test stimulus represents the front and back stimulus, respectively. (a) A schematic side view showing the manipulation of dynamic occlusion between each test stimulus and the background area. (b) A stereoscopic demonstration for uncrossed fusion. 

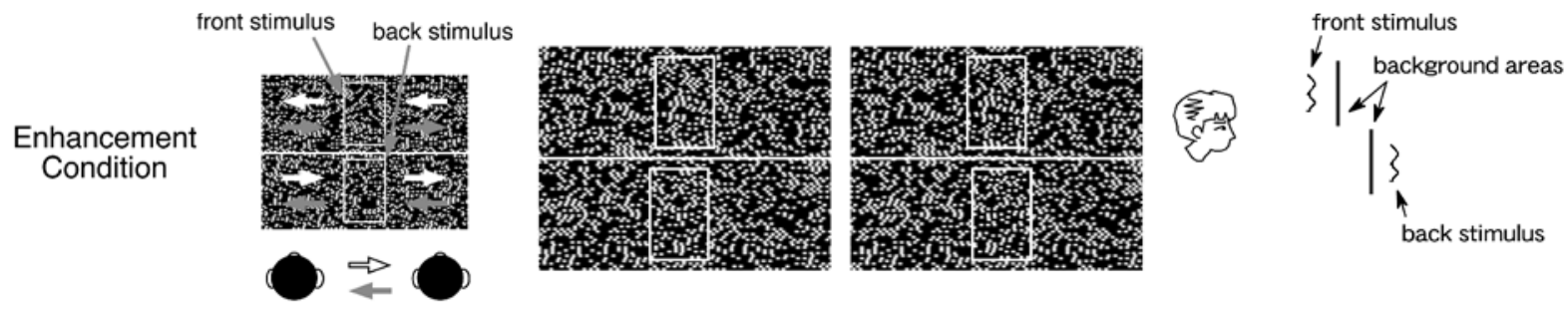

\section{No-Enhancement Condition}
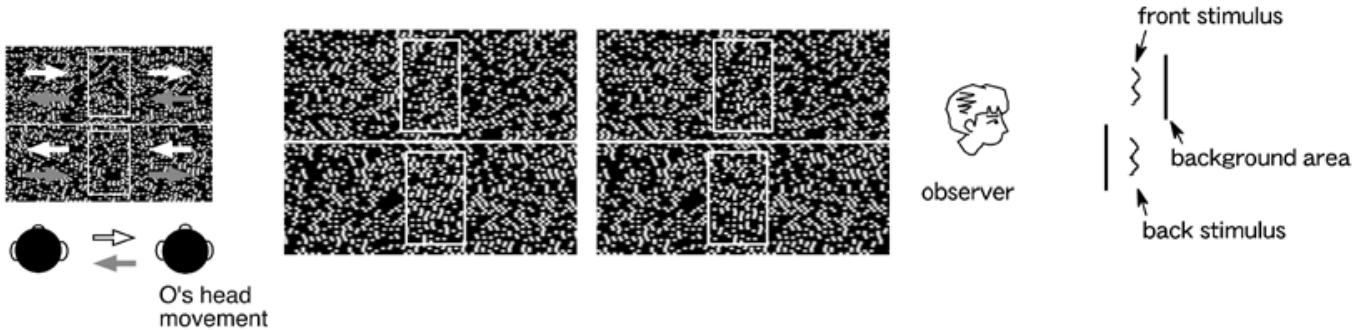

(a)

(b)

(c)

Figure 5. Illustrations demonstrating the stimulus conditions of enhancement (upper figures) and no-enhancement (lower figures) in Experiment 1. In all panels, the upper and lower test stimulus represents the front and back stimulus, respectively. (a) Schematic illustrations of the stimulus used in each condition. The arrows show the direction of the background motion relative to the observer's head movement. (b) Stereoscopic demonstrations of the stimulus displays for uncrossed fusion. (c) Schematic side view of the typical relative distance appearance of various components in the stimulus displays.

rounding the back stimulus. When viewed with lateral head movement, there did not appear to be any clear difference in the relative distances of the front and the back stimuli in this condition. Therefore, in this condition, which was named the no-enhancement condition, the background motion parallax canceled or did not enhance the difference in the relative distance between the front and back stimulus specified by the dynamic occlusion. Given that the relative distance cues cancel each other in this condition, no clear differences in perceived depth or perceived distance between the two test stimuli were expected. The finding of a high positive correlation between perceived depth and perceived distance, however, would support the idea that relative distance cues contribute to scaling parallactic depth by modifying information about absolute distance.

For each of the two stimulus conditions, there were two vertical arrangements of the test stimuli: the front stimulus on the top and the back stimulus on the bottom, and vice versa. Therefore, there were a total of four stimulus displays. All stimulus displays were void of fixation points.

Apparatus. The stimuli were presented on a 17-in. CRT monitor (MF8617E, IIYAMA) positioned $115.0 \mathrm{~cm}$ in front of a horizontal rail on which was mounted a moveable chinrest. The range of chinrest motion was limited to $10.0 \mathrm{~cm}$ by stoppers at both extremities. The position of the chinrest was detected by a potentiometer and was recorded by a personal computer (PC98, NEC) through an A/D board (AD12-16A, CONTEC).

The stimuli were predrawn and stored in a frame memory (VP1122, ASTRODESIGN) as 52 frames of $512 \times 240$ pixels, each with 8 bits gray scales. Presentation of the individual frames was under the control of the computer so that motions in the stimuli were yoked to the observers' head movements. The position of the observers' heads was sampled by the A/D board at a rate of $600 \mathrm{~Hz}$. This value was sufficiently higher than the CRT's refresh rate of $59.9 \mathrm{~Hz}$, and delays in switching frames after every $0.2 \mathrm{~cm}$ of head movement were less than about $1 / 60$ sec. All observers saw smooth dot motions in the stimulus displays with this setup.
Observers. Twenty volunteers, ranging in age from 21 to 35 years, were recruited from the National Institute of Bioscience and HumanTechnology community in Tsukuba City, Japan, to participate in this experiment. All of them had normal or corrected-to-normal vision. All but 1, who was the second author, were naive as to the purpose of the experiment.

Procedure. The experiment was conducted in a dimly lit laboratory room. The observers' task was to view the stimulus displays monocularly while actively moving their heads leftward and rightward, guided by the chinrest. They were instructed to report (1) which test stimulus (top or bottom) appeared to have greater depth and (2) which test stimulus (top or bottom) appeared more distant from them.

Before starting the experiment, the observers practiced moving their heads from side to side on the chinrest at a temporal frequency of $0.5 \mathrm{~Hz}$, and they familiarized themselves with the three-dimensional surfaces defined by the motion parallax displays. They were instructed to move their heads at $0.5 \mathrm{~Hz}$ or higher, because the magnitude of perceived depth from a given extent of motion parallax increases with head velocity and becomes constant with a maximum head velocity of $10.0 \mathrm{~cm} / \mathrm{sec}$ (H. Ono \& Ujike, 1993); this critical velocity is guaranteed by a temporal frequency of $0.5 \mathrm{~Hz}$ for a head movement extent of $10.0 \mathrm{~cm}$. The observers were also told to view the test stimuli for as long as they desired. In each trial, the experimenter verbally confirmed that the observers could see the three-dimensional corrugations in the two contoured test stimuli before they judged the depth and distance of the test stimuli. Each observer performed six trials for each of the four stimulus displays ( 2 stimulus conditions $\times 2$ arrangements); the first one of the six was treated as practice. The stimulus displays were presented in random order.

\section{Results}

Given that the only difference between the two test stimuli was relative distance information, any differences in the depth and/or distance judgments must be attributable to the 
manipulation of the relative distance cues. To determine the effect of the relative distance cues, depth preference ratios and distance preference ratios were calculated. The depth preference ratio was calculated by comparing the number of trials, from all observers, in which the back stimulus was judged to have greater depth than the front stimulus, with the total number of trials for each condition. Depth preference ratios deviating from chance level (.5) indicate that the relative distance cues influenced the perceived parallactic depth, and the greater the deviation from chance, the greater the influence of the relative distance cues. Moreover, depth preference ratios being greater or smaller than chance imply that, in many trials, the magnitude of depth perceived from the back stimulus was greater or smaller, respectively, than the front stimulus. Similarly, the distance preference ratios were calculated by comparing the number of trials, from all observers, in which the back stimulus was judged to be more distant than the front stimulus, with the total number of trials for each condition. As with the depth preference ratios, distance preference ratios deviating from chance level (.5) indicate that the relative distance cues influenced the absolute distance perception, and the greater the deviation, the greater the influence. Moreover, distance preference ratios being greater or smaller than chance imply that, in many trials, the perceived absolute distance to the back stimulus was further or nearer, respectively, than to the front stimulus.

The left and right pairs of bars in Figure 6 are the results from the enhancement and the no-enhancement conditions, respectively. The solid bars represent the depth preference ratios, and the striped bars represent the distance preference ratios; each bar shows the results of 200 trials ( 20 observers $\times 2$ vertical arrangements of the test stimuli $\times 5$ trials each). The depth preference ratios were significantly higher than chance level in the enhancement

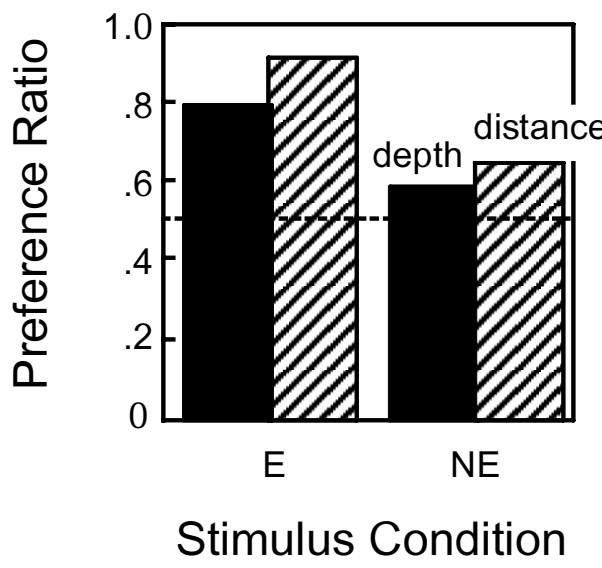

Figure 6. Ratios of trials in which the back stimulus was judged to have greater depth (depth preference ratio; solid bars) or to be more distant (distance preference ratio; striped bars) than the front stimulus in the enhancement $(E)$ and no-enhancement $(N E)$ conditions in Experiment 1. The horizontal dotted line represents chance level performance (i.e., .5). condition $\left[\chi^{2}(1, N=200)=60.5, p<.01\right]$ but not in the no-enhancement condition. Similarly, the distance preference ratios were significantly higher than chance level in the enhancement condition $\left[\chi^{2}(1, N=200)=124.8, p<\right.$ $.01]$ but not in the no-enhancement condition. Neither the depth nor the distance preference ratios were affected significantly by the vertical arrangement of the test stimuli.

In order to examine the relationship between the depth and the distance judgments more closely, the correlation between the two judgments was calculated for every trial and was expressed as an association index. The association index is a ratio calculated by comparing the number of trials, from all observers, in which the test stimulus that was judged to have greater depth was simultaneously judged to be more distant, with the total number of trials for each condition. An association index of unity indicates that the test stimulus perceived to have greater depth was always perceived as being more distant; an index of zero indicates that the test stimulus perceived to have the greater depth was always perceived as being the lesser distant; an index of .5 indicates no association between perceived depth and perceived distance. The association indices calculated from the data were 0.77 in the enhancement condition and 0.72 in the no-enhancement condition.

\section{Discussion}

The results from this experiment indicate that the relative distance cues of background motion parallax and dynamic occlusion contribute to scaling depth from motion parallax by modifying the information about the absolute distance of the test stimuli. In the enhancement condition, both the depth and the distance preference ratios were significantly greater than chance. In the no-enhancement condition, neither the depth nor the distance preference ratios deviated from chance. The association index in the noenhancement condition being as high as that in the enhancement condition, however, indicates that the test stimulus perceived to have greater depth was also perceived to be more distant.

As a consequence of the way in which we created the relative distance cue of dynamic occlusion (see the Method section), a third type of motion parallax (test-background motion parallax), which differed from both the test motion parallax and the background motion parallax, was introduced. Potentially, this test-background motion parallax could serve as a cue to the relative distance between each test stimulus and its surrounding background area. Our data do not support this idea, however, because the observers' perceptions agreed with what was expected from the dynamic occlusion information and not with what one might expect from the test-background motion parallax information. That is, in the enhancement condition, the dynamic occlusion cue specified the front stimulus as being in front of its background and the back stimulus as being behind its background. According to the background motion relative to the observers' head movements (see Figure 5a), however, the test-background motion parallax specified the front stimulus as being behind its back- 
ground and the back stimulus as being in front of its background. The predominant perception in this condition was that the back stimulus was farther away than the front stimulus. Given that the background motion parallax specified that the background area surrounding the front stimulus was in front of the background area surrounding the back stimulus in this condition, the reported perception is consistent with the information provided by dynamic occlusion and not with that provided by the test-background motion parallax (see the upper panel in Figure 5c). In the no-enhancement condition, the test-background motion parallax should not disturb the effect of the dynamic occlusion, because both cues provided consistent information about the relative distance order of the test stimuli with respect to their surrounding background areas. Although it has been suggested that relative distance perception is determined by the motions of various components in the display relative to the observers' head movements (Braunstein \& Tittle, 1988), this was not necessarily the case in the present experiment.

Our interpretation that dynamic occlusion dominated the test-background motion parallax in determining the perception of relative distance is consistent with H. Ono, Rogers, Ohmi, and M. E. Ono (1988). H. Ono et al. reported that when dynamic occlusion and motion parallax provide conflicting information about the depth order of surfaces, the perceived depth order is determined by dynamic occlusion if the depth separation is larger than 25.0 arc min in equivalent disparity. Although $\mathrm{H}$. Ono et al. expressed this critical depth separation in equivalent disparity, it is highly possible that the extent of depth separation itself is the critical variable. Given that the test-background motion parallax in our study simulated a relative distance separation of approximately $7.1 \mathrm{~cm}$, a value well above the 2.9$\mathrm{cm}$ separation corresponding to H. Ono et al.'s critical 25.0 arc min equivalent disparity separation, H. Ono et al.'s data are clearly consistent with the idea that, in our stimulus situation, perceived relative distance order was determined by dynamic occlusion rather than by the test-background motion parallax.

Before moving on to Experiment 2, two further comments about the results from Experiment 1 are in order. One pertains to why we chose to manipulate the relative distance cues of dynamic occlusion and background motion parallax in combination. The other pertains to the potential ramifications of a slight difference between the two test stimuli.

First, we manipulated dynamic occlusion and background motion parallax in combination for the following reason. In a preliminary experiment, we examined the effect of dynamic occlusion alone and failed to get a clear answer to the question of whether relative distance information contributes to the scaling of parallactic depth. In this preliminary experiment, the stimulus displays were the same as those in Experiment 1, except that the background area surrounding the test stimuli was not separated vertically and thus moved as a single unit. There were two stimulus conditions - namely, same and opposite - in which the di- rection of the entire background motion was either the same as or the opposite to the observers' head movements, respectively. We chose these two conditions because they produced a difference in the relative distance order of the background area relative to the test stimuli as specified by the motion parallax between them (the test-background motion parallax). Specifically, the background area was specified as being behind the test stimuli in the same condition, whereas it was specified as being in front of the test stimuli in the opposite condition. All other procedures were the same as those in Experiment 1. The results showed that, in the same condition, the back and the front stimuli were perceived to have the same magnitude of depth, whereas the back stimulus was perceived to be more distant than the front stimulus. On the other hand, in the opposite condition, the back stimulus was perceived to have greater depth and to be more distant. The association indices in the same and the opposite conditions were 0.46 and 0.81 , respectively. These results indicate that the direction of the background motion produced an asymmetry in the perception of depth but not in the perception of distance. This asymmetry suggests that unpredictable factors influenced the results when dynamic occlusion alone was manipulated. One plausible explanation of this result would be that the interaction between dynamic occlusion and test-background motion parallax affected the perception of depth but not the perception of distance. ${ }^{2}$ Thus, to control for this interaction, we combined the relative distance cue of background motion parallax with dynamic occlusion in Experiment 1. ${ }^{3}$

Second, the two test stimuli differed slightly in that the visible portion of the front (occluding) stimulus was fixed on the CRT and that of the back (occluded) stimulus moved in synchrony with the observers' head movements. On the basis of the following, however, it is our contention that this difference could not determine the depth judgments. The effect of the visible portions of the test stimuli's motion was examined in an additional experiment in which the experimental method was identical to that of Experiment 1, with the exception that the background area of the test stimuli was left blank and the test stimuli were not explicitly contoured. The visible portion of one of the two vertically arranged test stimuli moved in either the same (same condition) or the opposite (opposite condition) direction as the observers' head movements, whereas the average position of the random dots of these test stimuli remained stationary on the CRT (back stimulus). The visible portion of the other test stimulus, as well as the average position of the random dots, was fixed (front stimulus). The results indicated that the perceived depth of the front and the back stimuli did not differ in either condition. With respect to the perceived distance, in the same condition, the back stimulus was perceived as more distant than the front stimulus, whereas in the opposite condition, the two test stimuli were perceived as equally distant. ${ }^{4}$ The association indices in the same and the opposite conditions were .57 and .61, respectively. This pattern of results differs from 
those obtained in Experiment 1. In particular, the perceived depths of the two test stimuli in the condition in which the direction of the visible portion of the back stimulus, relative to the observers' heads, was the same as that in the enhancement condition (i.e., the same condition) did not differ. Therefore, it appears that the depth preference ratios reported in Experiment 1 were due to the combined contribution of dynamic occlusion and background motion parallax on the scaling of depth from the test motion parallax.

\section{EXPERIMENT 2}

Experiment 2 was designed to determine (1) whether relative distance cues other than those examined in Experiment 1 contribute to the scaling of parallactic depth and (2) the combined effects of multiple relative distance cues on the scaling of parallactic depth. The new relative distance cue introduced in this experiment was relative size, which provides information about the relative distances between two or more similar objects on the basis of their visual angles (Epstein \& Landauer, 1969; Higashiyama, 1979). In this experiment, the relative size of the two test stimuli was manipulated by reducing one of the test stim- uli relative to the other, without changing any other attributes of the test stimuli.

\section{Method}

Stimuli. There were two stimulus conditions, size and combination. In both conditions, the stimulus displays included two vertically arranged test stimuli that were composed of random dots similar to those in Experiment 1. Although the size of the test stimuli differed in this experiment, the shape and the extent of the test motion parallax within the test stimuli were the same as those in the previous experiment.

In the size condition, the relative size of the two test stimuli was manipulated (the upper panels in Figure 7). One test stimulus (defined as the front stimulus) was the same size as the test stimuli in the previous experiment, whereas the other test stimulus (defined as the back stimulus) was reduced in size by $4.0 \%$, to $4.6^{\circ} \times 3.0^{\circ}$. Accordingly, the spatial frequency of the two cycles of depth corrugation produced by the test motion parallax for the back stimulus was $0.44 \mathrm{cyc} / \mathrm{deg}$. In this condition, the background area surrounding the test stimuli was left blank, and the test stimuli were not explicitly contoured. For both test stimuli, the average position of the dots and the visible portion of random dots were fixed. In the combination condition, relative size was combined with the cues of dynamic occlusion and background motion parallax that were manipulated as those in the enhancement condition in Experiment 1 (the lower panels in Figure 7). These cues were combined so that they specified the same relative distance order of the test stimuli. The stimulus display in the

\section{Size Condition}
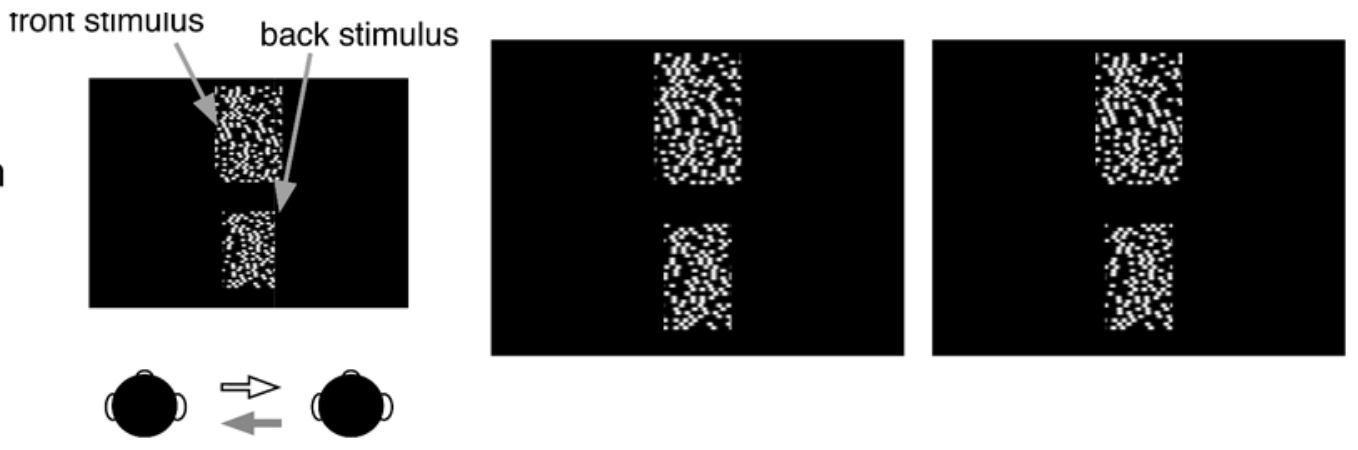

\section{Combination Condition}

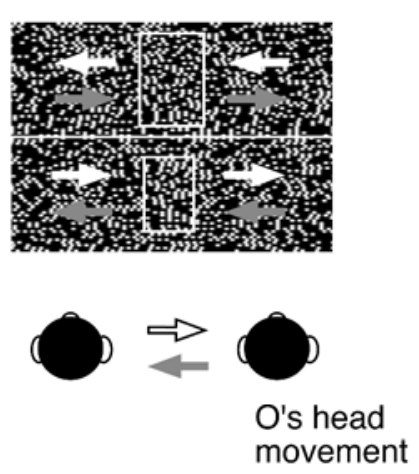

(a)
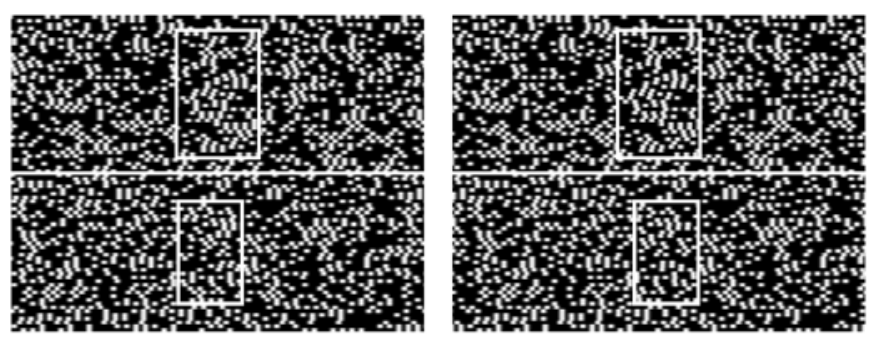

(b)

Figure 7. Illustrations demonstrating the stimulus conditions of size (upper figures) and combination (lower figures) in Experiment 2. In all panels, the upper and lower test stimuli represent the front and back stimuli, respectively. (a) Schematic illustrations of the stimulus display used in each condition. (b) Stereoscopic demonstrations of the stimulus displays for uncrossed fusion. 
combination condition was the same as that in the enhancement condition in Experiment 1, except that the size of the back stimulus was reduced by the same amount as in the size condition.

In both the size and the combination conditions, the vertical separation between the test stimuli was $1.1^{\circ}$. For each of the two stimulus conditions, there were two vertical arrangements of the test stimuli: the front stimulus on the top and the back stimulus on the bottom, and vice versa. Therefore, there was a total of four stimulus displays.

Observers. The observers were the same 20 volunteers who had participated in Experiment 1. All observers performed both of the stimulus conditions.

Procedure. The apparatus and the procedure were identical to those in the previous experiment. The observers viewed the stimulus display monocularly while actively moving their heads leftward and rightward, guided by the chinrest, at a temporal frequency of $0.5 \mathrm{~Hz}$ or higher. The observers were instructed to view the test stimuli for as long as they desired and to report (1) which stimulus (top or bottom) appeared to have greater depth and (2) which stimulus (top or bottom) appeared to be more distant. Each observer performed six trials for each of the four stimulus displays ( 2 stimulus conditions $\times 2$ arrangements); the first one of the six was treated as a practice trial. The stimulus displays were presented in random order.

\section{Results}

Depth and distance preference ratios were calculated as in Experiment 1. The left and right pairs of bars in Figure 8 are the results from the size and combination conditions, respectively. The solid bars represent the depth preference ratios, and the striped bars represent the distance preference ratios; each bar shows the results of 200 trials ( $20 \mathrm{ob}$ servers $\times 2$ vertical arrangements of the test stimuli $\times 5$ trials each). The depth preference ratios differed from chance (.5) in both the size condition $\left[\chi^{2}(1, N=200)=38.7, p<\right.$ $.01]$ and the combination condition $\left[\chi^{2}(1, N=200)=79.4\right.$, $p<.01]$. Likewise, the distance preference ratios differed from chance $(.5)$ in both the size condition $\left[\chi^{2}(1, N=200)=\right.$ $82.0, p<.01]$ and the combination condition $\left[\chi^{2}(1, N=\right.$ $200)=144.5, p<.01]$. Neither the depth nor the distance

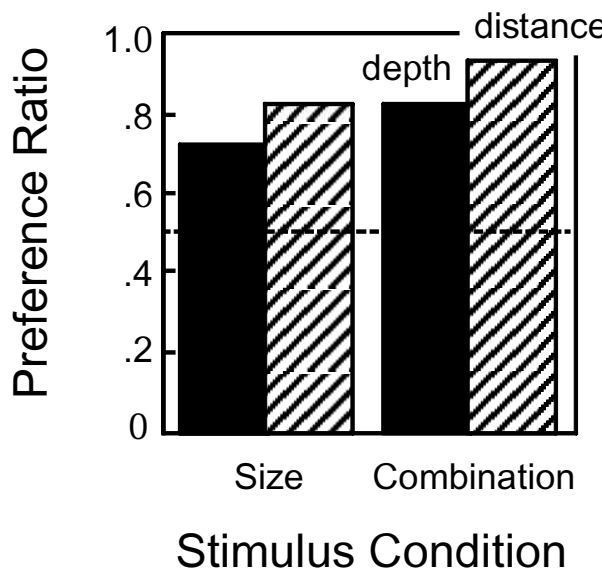

Figure 8. Ratios of trials in which the back stimulus was judged to have greater depth (depth preference ratio; solid bars) or to be more distant (distance preference ratio; striped bars) than the front stimulus in the size and combination conditions in Experiment 2 . The horizontal dotted line represents chance level performance (i.e., .5). preference ratios were affected significantly by the vertical arrangement of the test stimuli. As in Experiment 1, the correlation between the depth and distance judgments for every trial was examined by calculating the association index. This index was .74 in the size condition and .80 in the combination condition.

Finally, in order to examine the effect of the combination of relative distance cues, the results in the combination condition were compared with those in the size condition and with those in the enhancement condition of Experiment 1 . The $\chi^{2}$ tests showed that neither the depth nor the distance preference ratios in the combination condition differed significantly from those in the size condition or from those in the enhancement condition.

\section{Discussion}

Our results clearly show that the relative distance cue of relative size contributes to both the scaling of depth from motion parallax and the information about the absolute distance of visual objects. Moreover, the association index in the size condition indicates that relative size contributes to the scaling of parallactic depth by modifying the information about the absolute distance of the test stimuli. Although the two test stimuli differed in size, and consequently in the spatial frequency of the depth corrugations specified by the test motion parallax, this difference is not considered to have determined the difference in the perceived depth from the two test stimuli. This is so because Rogers and Graham (1982) reported that the sensitivity function of parallactic depth as a function of the spatial frequency of depth corrugations is U-shaped with peaks at $0.2-0.4$ cyc/deg. Given this, it is unlikely that the back stimulus ( 0.44 cyc/deg of the spatial frequency) was perceived as having greater depth than the front stimulus $(0.42$ cyc/deg) due to the spatial frequency properties of the visual system.

Both the depth and the distance preference ratios $(0.82$ and 0.93 , respectively) in the combination condition were higher than those in the size condition $(0.72$ and 0.82$)$ and those in the enhancement condition in Experiment $1(0.78$ and 0.90). Although these differences were not statistically significant, there is a clear tendency of larger preference ratios in the combination condition than in the other conditions. This result implies that the relative distance cues' effect on perceived absolute distance, and consequently, on scaling parallactic depth is enhanced.

\section{GENERAL DISCUSSION}

Our results show that relative distance cues contribute to the scaling of depth from motion parallax by modifying the information about the absolute distance of visual objects. Our results also show that when multiple relative distance cues, specifying the same relative distance direction, are combined with one another, their effects on the scaling of parallactic depth and on absolute distance information are enhanced. This combination effect is similar to that found with depth cues on depth perception (Bülthoff \& Mallot, 
1988; Curran \& Johnston, 1994; Johnston et al., 1994; Rogers \& Collett, 1989) and with absolute distance cues on distance perception(Künnapas, 1968; Swenson, 1932).

Our conclusion that relative distance cues affect absolute distance information is consistent with studies showing that estimations of perceived distance under reduced-cue conditions differ from those under full-cue conditions (Glennerster, Rogers, \& Bradshaw, 1998; Philbeck \& Loomis, 1997; Sedgwick, 1986). Under reduced-cue conditions, an isolated object is typically viewed in total darkness, and the main cues about the object's distance are the absolute distance cues of accommodation and/or vergence (Glennerster et al., 1998; Gogel, 1972). Under full-cue conditions, objects are typically viewed in an illuminated and structured environment, and, compared with reducedcue conditions, these conditions mainly add visual sources of information about the viewing environment such as relative distance cues (Gogel, 1972; Philbeck \& Loomis, 1997). It is well known that absolute distance estimations (Foley, 1977; Gogel, 1973; Künnapas, 1968; Philbeck \& Loomis, 1997; Tresilian, Mon-Williams, \& Kelly, 1999) and motor responses toward visual objects (Foley \& Held, 1972; Philbeck \& Loomis, 1997; Philbeck,Loomis, \& Beall, 1997) are more accurate in the full-cue conditions than in the reduced-cue conditions. These reports are in agreement with our finding that relative distance cues improve absolute distance information.

Obtaining absolute distance information from both absolute and relative distance cues is ecologically advantageous for the visual system. It is known that the absolute distance cues of accommodation and convergence, for example, are not necessarily very effective (Fisher \& Ciuffreda, 1988; Morrison \& Whiteside, 1984; Richards \& Miller, 1969). Particularly, it has been argued that the effectiveness of these cues decreases at viewing distances of greater than about 1 or 2 meters (Komoda \& Ono, 1974; Leibowitz \& Moore, 1966; Leibowitz, Shiina, \& Hennessy, 1972; Wallach \& Floor, 1971). On the other hand, some relative distance cues, such as occlusion, relative size, and aerial perspective, can provide effective distance information at even greater viewing distances (Cutting \& Vishton, 1995). Therefore, assisted by relative distance cues, absolute distance information can be more accurate over a wider range of viewing distances.

Our finding that relative distance cues contribute to the scaling of parallactic depth is consistent with the recent report that parallactic depth perception in reduced-cue conditions differs from that in full-cue conditions (Ohtsuka, Ujike, \& Saida, 1999). Ohtsuka et al. presented observers with stimuli containing constant extents of motion parallax at various viewing distances $(80-240 \mathrm{~cm})$ and found the following relationship between the magnitude of perceived depth and the viewing distance. In the full-cue condition, the magnitude of perceived depth increased as predicted geometrically on the basis of viewing distance. In the reduced-cue condition, the magnitude of perceived depth was relatively unaffected by viewing distance. Thus, as with absolute distance estimation, these results suggest that relative distance cues increase the accuracy of parallactic depth perception.

Absolute distance information is used not only for scaling depth information from motion parallax but also for scaling depth information from binocular disparity (Howard \& Rogers, 1995; H. Ono \& Comerford, 1977). Thus, it is likely that relative distance cues contribute to this scaling. In support of this idea, Glennerster et al. (1998) reported that the magnitude of perceived stereoscopic depth as a function of viewing distance $(38-228 \mathrm{~cm})$ is different in full-cue conditions than it is in reduced-cue conditions. Specifically, they reported that the magnitude of perceived depth more closely approximated the geometrical prediction based on the viewing distance in the full-cue condition than in the reduced-cue condition; the result was similar to that reported by Ohtsuka et al. (1999) with respect to parallactic depth.

Although it has been argued that parallactic depth is scaled primarily by perceived absolute distance (Rivest, Ono, \& Saida, 1989), our results are not consistent with this argument. Namely, the association indices obtained in our experiments ranged from .72 to .80 , indicating a nonperfect correlation. In addition, we found, in some conditions, that the indices were lower and close to .5, indicating no correlation, and/or that the distance preference ratio deviated from chance, whereas the depth preference ratio did not (in the preliminary experiment and in the additional experiment described in the Discussion section in Experiment 1). These results suggest that scaling parallactic depth does not necessarily depend on perceived distance. This interpretation is consistent with studies of binocular stereopsis. For example, Brenner and Landy (1999) simultaneously measured perceived depth and perceived distance from binocularly disparate stimuli and found that the distance information used for scaling depth and for perceiving distance differed. A similar discussion has been presented in previous studies (Epstein, 1973; O'Leary \& Wallach, 1980; H. Ono \& Comerford, 1977; Wallach, Gillam, \& Cardillo, 1979) in which the distance information used for scaling, typically termed registered distance, is distinguished from perceived distance. Thus, it can be said that the distance information used for the perception of distance is different than the information used for scaling depth from motion parallax, as well as from binocular disparity.

\section{REFERENCES}

Andersen, G. J., \& Braunstein, M. L. (1983). Dynamic occlusion in the perception of rotation in depth. Perception \& Psychophysics, 34, 356-362.

Braunstein, M. L., \& Tittle, J. S. (1988). The observer-relative velocity field as the basis for effective motion parallax. Journal of Experimental Psychology: Human Perception \& Performance, 14, 582-590.

BRENNER, E., \& LANDY, M. S. (1999). Interaction between the perceived shape of two objects. Vision Research, 39, 3834-3848.

Bülthoff, H. H., \& Mallot, H. A. (1988). Integration of depth modules: Stereo and shading. Journal of the Optical Society of America A, 5, 1749-1758.

Curran, W., \& Johnston, A. (1994). Integration of shading and texture cues: Testing the linear model. Vision Research, 34, 1863-1874.

Cutting, J. E., \& Vishton, P. M. (1995). Perceiving layout and knowing 
distances: The integration, relative potency, and contextual use of different information about depth. In W. Epstein \& S. Rogers (Eds.), Perception of space andmotion (pp. 69-117). San Diego: Academic Press.

EPSTEIN, W. (1973). The process of "taking-into-account" in visual perception. Perception, 2, 267-285.

Epstein, W., \& Landauer, A. A. (1969). Size and distance judgments under reduced conditions of viewing. Perception \& Psychophysics, $\mathbf{6}$, 269-272.

Fisher, S. K., \& Ciuffreda, K. J. (1988). Accommodation and apparent distance. Perception, 17, 609-621.

Foley, J. M. (1977). Effect of distance information and range on two indices of visually perceived distance. Perception, 6, 449-460.

Foley, J. M., \& HeLD, R. (1972). Visually directed pointing as a function of target distance, direction, and available cues. Perception \& Psychophysics, 12, 263-268.

Gillam, B. (1995). The perception of spatial layout from static optical information. In W. Epstein \& S. Rogers (Eds.), Perception of space and motion (pp. 23-67). San Diego: Academic Press.

Glennerster, A., Rogers, B. J., \& Bradshaw, M. F. (1998). Cues to viewing distance for stereoscopic depth constancy. Perception, 27, 1357-1365.

Gogel, W. C. (1972). Scalar perceptions with binocular cues of distance. American Journal of Psychology, 85, 477-498.

Gogel, W. C. (1973). The organization of perceived space: I. Perceptual interactions. Psychologische Forschung, 36, 195-221.

Higashiyama, A. (1979). The perception of size and distance under monocular observation. Perception \& Psychophysics, 26, 230-234.

Howard, I. P., \& Rogers, B. J. (1995). Binocular vision and stereopsis. New York: Oxford University Press.

Johnston, E. B., Cumming, B. G., \& Landy, M. S. (1994). Integration of stereopsis and motion shape cues. Vision Research, 34, 2259-2275.

Kaplan, G. A. (1969). Kinetic disruption of optical texture: The perception of depth at an edge. Perception \& Psychophysics, 6, 193-198.

Komoda, M. K., \& ONO, H. (1974). Oculomotor adjustments and size-distance perception. Perception \& Psychophysics, 15, 353-360.

KÜNNAPAS, T. (1968). Distance perception as a function of available visual cues. Journal of Experimental Psychology, 77, 523-529.

Leibowitz, H. [W.], \& Moore, D. (1966). Role of changes in accommodation and convergence in the perception of size. Journal of the Optical Society of America, 56, 1120-1129.

Leibowitz, H. W., ShIINA, K., \& HenNessy, R. T. (1972). Oculomotor adjustments and size constancy. Perception \& Psychophysics, 12, 497-500.

Morrison, J. D., \& Whiteside, T. C. D. (1984). Binocular cues in the perception of distance of a point source of light. Perception, 13, 555-566.

OHTSUKa, S., UJIKe, H., \& SAIDA, S. (1999). Scaling of motion parallax and absolute distance perception. Japanese Journal of Psychonomic Science, 17, 100-110. [in Japanese]

O'Leary, A., \& Wallach, H. (1980). Familiar size and linear perspective as distance cues in stereoscopic depth constancy. Perception \& Psychophysics, 27, 131-135.

ONo, H. \& Comerford, J. (1977). Stereoscopic depth constancy. In W. Epstein (Ed.), Stability and constancy in visual perception: Mechanisms and process (pp. 91-128). New York: Wiley.

Ono, H., Rogers, B. J., Ohmi, M., \& Ono, M. E. (1988). Dynamic occlusion and motion parallax in depth perception. Perception, 17, 255-266.

ONO, H., \& UJIKE, H. (1993). Equal depth contour as a function of different velocities of head movement. Perception, 22 (Suppl.), 86.

Ono, M. E., RIvest, J., \& ONo, H. (1986). Depth perception as a function of motion parallax and absolute-distance information. Journal of Experimental Psychology: Human Perception \& Performance, 12, 331-337.

Philbeck, J. W., \& LoOMIS, J. M. (1997). Comparison of two indicators of perceived egocentric distance under full-cue and reduced-cue conditions. Journal of Experimental Psychology: Human Perception \& Performance, 23, 72-85.

Philbeck, J. W., Loomis, J. M., \& BeAll, A. C. (1997). Visually perceived location is an invariant in the control of action. Perception \& Psychophysics, 59, 601-612.

Richards, W., \& Miller, J. F., JR. (1969). Convergence as a cue to depth. Perception \& Psychophysics, 5, 317-320.

Rivest, J., Ono, H., \& SAIDA, S. (1989). The roles of convergence and apparent distance in depth constancy with motion parallax. Perception \& Psychophysics, 46, 401-408.

Rogers, B. J., \& Collett, T. S. (1989). The appearance of surfaces specified by motion parallax and binocular disparity. Quarterly Journal of Experimental Psychology, 41A, 697-717.

Rogers, B. [J.], \& Graham, M. [E.] (1979). Motion parallax as an independent cue for depth perception. Perception, 8, 125-134.

Rogers, B. J., \& Graham, M. E. (1982). Similarities between motion parallax and stereopsis in human depth perception. Vision Research, 22, 261-270.

Sedgwick, H. A. (1986). Space perception. In K. R. Boff, L. Kaufman, \& J. P. Thomas (Eds.), Handbook of perception and human performance: Vol. I. Sensory processes and perception (pp. 21-1 to 21-57). New York: Wiley.

SWEnson, H. A. (1932). The relative influence of accommodation and convergence in the judgment of distance. Journal of General Psychology, 7, 360-380.

Tresilian, J. R., Mon-Williams, M., \& Kelly, B. M. (1999). Increasing confidence in vergence as a cue to distance. Proceedings of the Royal Society of London: Series B, 266, 39-44.

van Damme, W. J. M., OOST ERhoff, F. H., \& VAN DE Grind, W. A. (1994). Discrimination of 3-D shape and 3-D curvature from motion in active vision. Perception \& Psychophysics, 55, 340-349.

Wallach, H. \& Floor, L. (1971). The use of size matching to demonstrate the effectiveness of accommodation and convergence as cues for distance. Perception \& Psychophysics, 10, 423-428.

Wallach, H. Glllam, B., \& Cardillo,L. (1979). Some consequences of stereoscopic depth constancy. Perception \& Psychophysics, 26, 235-240.

\section{NOTES}

1. In the present study, absolute distance is used to indicate the extent of spatial separation from the observer to an object. Relative distance is used to refer to the spatial separation between the objects and is usually referred to as depth. The magnitude of the relative distance or of the depth is the extent of the separation.

2. Dynamic occlusion and test-background motion parallax might interact as follows. In the same condition, the information provided by the two cues conflict for the back stimulus and agree for the front stimulus. On the contrary, in the opposite condition, the information provided by the two cues conflict for the front stimulus and agree for the back stimulus. If it is assumed that this cue conflict inhibits depth perception from the test motion parallax, our results are explained well.

3 . In the enhancement condition, the cues of dynamic occlusion and test-background motion parallax conflicted for both the front and the back stimuli. On the other hand, the cues agreed for both of the test stimuli in the no-enhancement condition.

4. The observed difference in the result of the distance perception between the same and the opposite conditions may have been caused by a well-known asymmetry in depth perception: Objects displayed on a screen, two-dimensionally, seldom appear to be in front of the screen, with manipulations of depth cues, whereas they easily appear to be behind the screen. In the experiment, motion parallax between the frame of the monitor and the moving visible portion of the back stimulus specified the moving portion as being in front of the monitor in the opposite condition and as being behind it in the same condition. Therefore, the back stimulus might easily appear to be behind the monitor and, thus, also to be behind the front stimulus in the same condition; this agrees with our result.

(Manuscript received March 19, 1998; revision accepted for publication July 10, 2001.) 